\title{
Brain Lobectomy
}

National Cancer Institute

\section{Source}

National Cancer Institute. Brain Lobectomy. NCI Thesaurus. Code C52004.

Surgical removal of a lobe of the brain. 